\title{
Fractional charge excitations in fermionic ladders.
}

\author{
B.N. Narozhny, ${ }^{1}$ S.T. Carr, ${ }^{1}$ and A.A. Nersesyan ${ }^{1,2}$ \\ ${ }^{1}$ The Abdus Salam ICTP, Strada Costiera 11, Trieste, 34100, Italy. \\ ${ }^{2}$ The Andronikashvili Institute of Physics, Tamarashvili 6, 017r, Tbilisi, Georgia
}

(Dated: November 6, 2018)

\begin{abstract}
The system of interacting spinless fermions hopping on a two-leg ladder in the presence of an external magnetic field is shown to possess a long range order: the bond density wave or the staggered flux phase. In both cases the elementary excitations are $Z_{2}$ kinks and carry one half the charge of an electron.

PACS numbers: 71.10.Pm; 71.30.+h
\end{abstract}

It has long been established both theoretically [1, 2] and experimentally $[\underline{3}$ ] that quantum numbers of elementary excitations in interacting many-body systems are not necessarily limited to the values characterizing free particles. While excitations carrying, for example, an electric charge double the free electron charge $e$ can be visualized as pairs of the original particles [4], the fractionally charged excitations may appear counterintuitive $[2,5,6]$. By far the most celebrated example of such excitations are the fractionally charged quasiparticles in the quantum Hall state [5]. At about the same time the notion of fractional charge appeared in the Peierls model applied to quasi-one-dimensional conducting polymers [2]. More recently 6], fractionally charged excitations were predicted in the context of the extended Hubbard model.

In this paper we argue that charge fractionalization is a generic phenomenon in the sense that it appears already in the simplest model of spinless fermions in a magnetic field. Restricting the fermions to hop on a two-leg ladder [7, 8], we show that just nearest-neighbor interaction (if it is strong enough) leads to long range order (LRO) with a doubly degenerate ground state. The elementary excitations are then quantum domain walls or $Z_{2}$ kinks that carry the charge $e / 2$.

The applied field plays a crucial and somewhat surprising role in the problem. In the absence of the field the only possible LRO is the bond density wave (BDW) similar to that of Ref. [6. However in the presence of the field different types of LRO are possible. The most spectacular manifestation of the field is the staggered flux phase [9, 10] illustrated in Fig.11 Note that the staggered order parameter here is induced by a uniform field [11].

To build some intuition about the ordered phases consider the strong coupling limit of the model where the transverse interaction $V_{\perp}$ is the strongest, so that no rung of the ladder can be doubly occupied. For the quarterfilled ladder the starting point is a state where there is one electron per two plaquettes. The hopping terms tend to delocalize the electrons along the links. If the longitudinal interaction is repulsive $V_{\|}>0$, then the most favorable configuration is that in which the electrons, avoiding to reside on neighboring sites of the same chain, get delo-

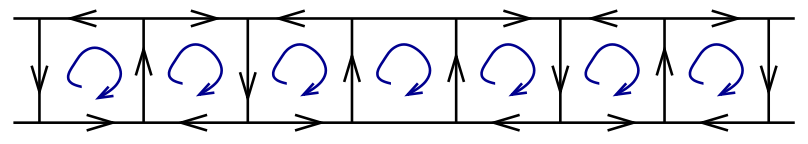

FIG. 1: The staggered flux phase in the two-leg ladder penetrated by a magnetic field. The arrows inside the plaquettes indicate the direction of the applied uniform field (directed inside the plane of the Figure). The arrows along the links indicate directions of the staggered currents. The central plaquette is the domain wall between the two degenerate ground states. This excitation carries the charge $e / 2$.

calized on rungs thus leading to a commensurate BDW. If, on the other hand, $V_{\|}<0$ then the system has a natural tendency to phase separate which is opposed by the flux. If the flux is strong enough the electrons are placed on every other plaquette and are completely delocalized around them (thus avoiding double occupancy of the rungs) producing currents circulating in the same direction (determined by the field). This can be viewed as a staggered current superimposed upon the average, uniform (persistent) current.

In this paper we show how the above cartoon picture arises starting from the weak coupling limit. Our strategy is the following: (i) we diagonalize the Hamiltonian in the absence of interaction; (ii) linearize the exact spectrum near the Fermi points and bosonize the model; (iii) finally, we solve the bosonic problem and find the ground state of the interacting system.

The single-particle spectrum of the problem consists of two one-dimensional bands and is governed by the magnetic flux through a plaquette in units of the flux quantum $f$ as well as the ratio of the transverse and longitudinal hopping amplitudes $\tau$. It is possible to partially fill only one band and have only two Fermi points. In this case the low energy physics of the problem is similar to that of the XXZ spin chain [12]. If the interaction is weak, then the system is in the Luttinger liquid regime (i.e. similar to the gapless phase of the XXZ chain). If, on the other hand the two Fermi points are commensurate with the lattice and the interaction is sufficiently strong, then the umklapp scattering becomes relevant in 
the renormalization group sense and opens a gap. The ground state of this gapful phase possesses LRO and is doubly degenerate. Now, due to the applied field, the physics of the ladder is richer than that of the single chain. Depending on the sign of the umklapp term different types of LRO are possible: BDW in the repulsive case, or the staggered flux phase [9] (or an orbital antiferromagnet [10]) in the case of attraction.

Having sketched our line of reasoning we now describe our calculations. In this paper we focus on the particular parameter regimes where the system exhibits the fractional charge. Mathematical details and a description of the full phase diagram of the system as well as a quantitative description of the strong coupling limit will be discussed elsewhere 13].

We start with the tight-binding Hamiltonian

$$
\begin{aligned}
\mathcal{H}=- & \sum_{n}\left[\frac{1}{2} \sum_{i=1,2}\left(t_{||}\left(y_{i}\right) c_{i}^{\dagger}\left(x_{n}\right) c_{i}\left(x_{n+1}\right)+\text { h.c. }\right)\right. \\
& \left.+t_{\perp} c_{1}^{\dagger}\left(x_{n}\right) c_{2}\left(x_{n}\right)+\text { h.c. }\right]+\mathcal{H}_{i n t},
\end{aligned}
$$

where $c_{j}\left(x_{n}\right)$ is the electron annihilation operator on the chain $j$ at the site $x_{n}, t_{\perp}$ and $t_{\|}$are the transverse and the longitudinal hopping amplitudes. The magnetic field $B$ is introduced by means of the Peierls substitution [14]. Choosing the Landau gauge 15. with the vector potential $\mathbf{A}=B(-y, 0,0)$ and defining the $y$ coordinates of the chains as $y_{1(2)}= \pm b / 2$ we write the longitudinal hopping amplitude as

$$
t_{\|}(y)=t_{\|}^{(0)} e^{2 \pi i f y / b}
$$

where $f=B a b / \phi_{0}, \phi_{0}$ being the flux quantum and $a$ the lattice spacing along the chains. The control parameter $\tau$ is defined as $\tau=t_{\perp} / t_{\|}^{(0)} ; t_{\|}^{(0)}$ plays the role of the bandwidth and hereafter will be set to unity. Expressed in terms of the flux the model is explicitly gauge invariant.

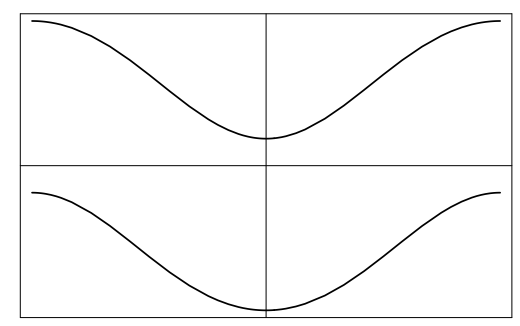

FIG. 2: The spectrum in the region $\tau>1, \tau \cos \pi f>\sin ^{2} \pi f$.

The physical quantities we shall study in this paper are the electrical current and particle density. In terms of the lattice operators $c_{j}\left(x_{n}\right)$ the in-chain current is given by

$$
J_{1(2)}=-\frac{i}{2}\left[c_{1(2)}^{\dagger}\left(x_{n}\right) c_{1(2)}\left(x_{n+1}\right) e^{\mp \pi i f}-h . c .\right],
$$

while the transverse current is defined as

$$
J_{\perp}=-i \tau\left[c_{1}^{\dagger}\left(x_{n}\right) c_{2}\left(x_{n}\right)-h . c .\right] \text {. }
$$

Similarly, the bond density on transverse links is

$$
\rho_{\perp}=c_{1}^{\dagger}\left(x_{n}\right) c_{2}\left(x_{n}\right)+\text { h.c. }
$$

In the absence of interaction the Hamiltonian Eq. (1) can be diagonalized with the help of the linear transformation

$$
c_{1}(q)=u_{q} \alpha_{q}+v_{q} \beta_{q} ; \quad c_{2}(q)=v_{q} \alpha_{q}-u_{q} \beta_{q}
$$

where the "coherence factors" are (the signs are explicit in Eq.(6) so that the coherence factors are positive)

$$
u_{q}^{2}\left(v_{q}^{2}\right)=\frac{1}{2}\left[1 \mp \frac{\sin q \sin \pi f}{\sqrt{\sin ^{2} q \sin ^{2} \pi f+\tau^{2}}}\right] .
$$

The exact spectrum of the system consists of two onedimensional bands

$$
\epsilon_{\alpha(\beta)}(k)=-\cos k \cos \pi f \mp \sqrt{\sin ^{2} k \sin ^{2} \pi f+\tau^{2}},
$$

where $k$ in the momentum along the chains. Since the transformation $f \rightarrow 1-f$ preserves the spectrum we only need to consider the fluxes such that $0 \leq f \leq 1 / 2$. If the transverse hopping amplitude is large enough so that $\tau>\cos \pi f$ the spectrum possesses a band gap and resembles a band insulator, see Fig. 22 If the flux is not too small, $\sin ^{2} \pi f>\tau \cos \pi f$, the bands acquire a doublewell shape shown in Fig. 3

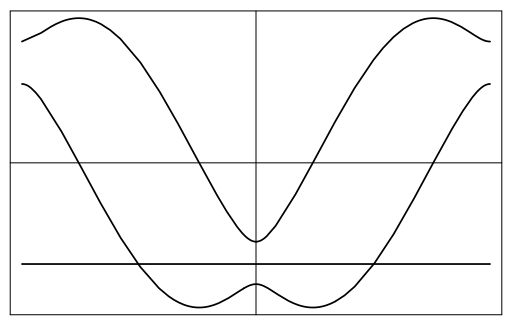

FIG. 3: The spectrum in the region $(\sin \pi f)^{2} / \tau>\cos \pi f>\tau$. The horizontal line depicts the chemical potential such that there are only two Fermi points.

Now we focus on the situation where the lower band is only partially filled (the upper band is empty) and there are only two Fermi points. The ground state in the absence of interaction is characterized by the persistent current flowing along the chains. Indeed, the ground state expectation value of the transverse current Eq. (4) vanishes $\left\langle J_{\perp}\right\rangle=0$, while the in-chain current Eq. (3) is non-zero as long as the flux is applied

$$
\left\langle J_{1}\right\rangle=\frac{\sin \pi f}{2} \sum_{q=-k_{F}}^{k_{F}}\left[\frac{\sin ^{2} q \cos \pi f}{\sqrt{\sin ^{2} q \sin ^{2} \pi f+\tau^{2}}}-\cos q\right] \text {. }
$$

The current flows along the two chains in opposite directions $\left\langle J_{1}\right\rangle=-\left\langle J_{2}\right\rangle$, so that the net current is equal 
to zero as it should. The Fermi momentum in Eq. (9) depends on the applied field as

$$
\cos k_{F}=-\tilde{\mu} \cos \pi f-\sqrt{\tau^{2}+\left(1-\tilde{\mu}^{2}\right) \sin ^{2} \pi f},
$$

where $\tilde{\mu}$ is the chemical potential in units of $t_{\|}^{(0)}$.

It is noteworthy that all (filled) states contribute to the persistent current Eq. (9). The persistent current in this problem is not an infrared phenomenon and thus can not be accounted for by an effective low energy theory. The low energy effects we discuss below are thus taking place on the background of this single-particle persistent current. We will not discuss further corrections to Eq. (9) in this paper.

To formulate an effective low energy theory we now linearize the exact spectrum in the vicinity of the Fermi points Eq. (10), assuming that that the $k=0$ features of the exact spectrum are far away from the Fermi level, the condition that has to be checked later. Since only one band is filled, this step is fairly standard and is similar to the case of a single chain 12. Separating fast and slow variables, we introduce the left- and right-movers for the lower band and find the standard kinetic term

$$
\mathcal{H}_{0}=v_{F}^{\alpha} \sum_{k} k\left(\alpha_{R}^{\dagger}(k) \alpha_{R}(k)-\alpha_{L}^{\dagger}(k) \alpha_{L}(k)\right),
$$

where the Fermi velocity is

$$
v_{F}^{\alpha}=\sin k_{F} \frac{\sqrt{\left(1-\tilde{\mu}^{2}\right) \sin ^{2} \pi f+\tau^{2}}}{\sqrt{\sin ^{2} k_{F} \sin ^{2} \pi f+\tau^{2}}} .
$$

To obtain the final form of the effective theory we need to express the physical observables in terms of the low energy fields (in doing so we approximate the coherence factors by their values at the Fermi points $u_{0}$ and $v_{0}$ ). The smooth part of the particle density is then given by

$$
\rho_{1}=u_{0}^{2} J_{R}+v_{0}^{2} J_{L} ; \quad \rho_{2}=v_{0}^{2} J_{R}+u_{0}^{2} J_{L},
$$

where $J_{R(L)}=: \alpha_{R(L)}^{\dagger} \alpha_{R(L)}$ :. Mostly we shall be interested in staggered operators with expectation values vanishing in the absence of interaction. The operators of interest are the staggered currents (defined on links)

$$
\begin{gathered}
j_{1(2)}\left(x_{n}, x_{n+1}\right)=\frac{(-1)^{n}}{2} u_{0} v_{0}\left(\alpha_{L}^{\dagger}\left(x_{n}\right) \alpha_{R}\left(x_{n+1}\right) e^{-i \pi f}\right. \\
\left.-\alpha_{L}^{\dagger}\left(x_{n+1}\right) \alpha_{R}\left(x_{n}\right) e^{i \pi f}-h . c .\right), \\
j_{\perp}\left(x_{n}\right)=i t_{\perp}(-1)^{n}\left(v_{0}^{2}-u_{0}^{2}\right)\left(\alpha_{L}^{\dagger}\left(x_{n}\right) \alpha_{R}\left(x_{n}\right)-\text { h.c. }\right),
\end{gathered}
$$

and the staggered bond density

$$
\rho_{\perp}^{(s)}\left(x_{n}\right)=(-1)^{n}\left(\alpha_{L}^{\dagger}\left(x_{n}\right) \alpha_{R}\left(x_{n}\right)+h . c\right) .
$$

Current conservation requires $\left|\left\langle j_{\perp}\right\rangle\right|=2\left|\left\langle j_{1(2)}\right\rangle\right|$.

We now turn to the discussion of interaction effects. For simplicity we consider the nearest-neighbor densitydensity interaction

$$
\begin{gathered}
\mathcal{H}_{\text {int }}=\sum_{n}\left[V_{\text {॥ }}\left(n_{1}\left(x_{n}\right) n_{1}\left(x_{n+1}\right)+n_{2}\left(x_{n}\right) n_{2}\left(x_{n+1}\right)\right)\right. \\
\left.+V_{\perp} n_{1}\left(x_{n}\right) n_{2}\left(x_{n}\right)\right],
\end{gathered}
$$

with $n_{j}=: c_{j}^{\dagger} c_{j}$ : (colons indicate normal ordering).

In terms of left- and right-movers (neglecting trivial renormalizations of the Fermi velocity) we rewrite the interaction Eq. (16) in position space as

$$
\begin{aligned}
& \mathcal{H}_{\text {int }} \approx \sum_{i}\left[g_{1}: \alpha_{R}^{\dagger}\left(x_{i}\right) \alpha_{R}\left(x_{i}\right) \alpha_{L}^{\dagger}\left(x_{i}\right) \alpha_{L}\left(x_{i}\right):\right. \\
&\left.\quad+\left(g_{2}: \alpha_{R}^{\dagger}\left(x_{i}\right) \alpha_{R}^{\dagger}\left(x_{i+1}\right) \alpha_{L}\left(x_{i}\right) \alpha_{L}\left(x_{i+1}\right):+ \text { h.c. }\right)\right]
\end{aligned}
$$

where the new interaction constants are 16 ]

$$
g_{1}=2 V_{\|} u_{0}^{2} v_{0}^{2}\left(1-\cos 2 k_{F}\right)+V_{\perp}\left(u_{0}^{2}-v_{0}^{2}\right)^{2} ;
$$

$$
g_{2}=-2 V_{\|} e^{2 i k_{F}} u_{0}^{2} v_{0}^{2} .
$$

The first term in Eq. (17) is the density-density interaction that can be taken into account exactly by means of bosonization. The second term is the umklapp interaction that does not conserve momentum and thus is only relevant when $k_{F}$ is commensurate with the lattice (i.e. for the quarter-filled ladder).

In the commensurate case the Fermi momentum $k_{F}=$ $\pi / 2$ and the chemical potential $\tilde{\mu}=-\sqrt{\sin ^{2} \pi f+\tau^{2}}$. This is consistent with the requirement of having only two Fermi points when $2 \tau>|\cos 2 \pi f| / \cos \pi f$. In this case the combinations of coherence factors in Eqs. (17) and (14) simplify to

$$
u_{0}^{2}-v_{0}^{2}=(\sin \pi f) / \tilde{\mu} \quad ; \quad u_{0} v_{0}=\tau / \tilde{\mu} .
$$

Now we use the standard bosonization procedure and express the interaction Hamiltonian Eq. (17) in terms of a bosonic field $\phi$ and the conjugate momentum $\Pi$. Combining it with the kinetic term we arrive to the usual form of the Luttinger liquid Hamiltonian (here $\alpha$ is the bosonic cut-off)

$$
\mathcal{H}=\frac{v_{F}^{\alpha}}{2}\left[K \Pi^{2}+\frac{1}{K}\left(\partial_{x} \phi\right)^{2}\right]-\frac{g_{2}}{2 \pi^{2} \alpha^{2}} \cos \sqrt{16 \pi} \phi .
$$

where the Luttinger liquid parameter $K$ is determined by the interaction constant Eq. (18a) as $K=[(1-$ $\left.\left.g_{1} / 2 \pi v_{F}^{\alpha}\right) /\left(1+g_{1} / 2 \pi v_{F}^{\alpha}\right)\right]^{1 / 2}$.

The behavior of the effective theory Eq. (20) is well known [12]. At the critical value of the Luttinger liquid 
parameter $K=1 / 2$ [17] the system exhibits a BerezinskiKosterlitz-Thouless (BKT) 18] transition to a gapful phase with broken $Z_{2}$ symmetry and LRO. The type of ordering depends on the sign of the coupling constant $g_{2}$ :

(i) If the umklapp term is repulsive $g_{2}>0$ then the local minima of the potential are achieved at $\phi=$ $\sqrt{\pi / 16}+n \sqrt{\pi / 4}, n=0, \pm 1, \ldots$ In this case the staggered bond density Eq. (15) $\rho_{\perp}^{(s)} \sim \sin \sqrt{4 \pi} \phi$ acquires a non-zero expectation value and LRO is of the BDW-type. This ordering resembles the charge density wave in the extended Hubbard model [6] at 1/4 filling.

(ii) If, on the other hand, $g_{2}<0$ [19], then the local minima are at $\phi=n \sqrt{\pi / 4}$ and now there exists the staggered current Eq. (14) $j_{\perp} \sim \cos \sqrt{4 \pi} \phi$ leading to the staggered flux phase illustrated in Fig. 10 Clearly, the currents are non-zero only in the presence of the applied field, as $j_{\perp} \propto \sin \pi f$. This is a surprise: we find that the uniform field causes the staggered current.

In both cases the ground state is doubly degenerate and the elementary excitation is a "quantum domain wall" or, in terms of the effective theory Eq. (20), a $Z_{2}$ kink interpolating between the two neighboring minima. The charge carried by the kink is related to the distance $\Delta \phi$ between the minima [as follows from Eq. [13)]

$$
Q=e \frac{\Delta \phi}{\sqrt{\pi}}
$$

Since both types of ordering originate from the same interaction term in Eq. (20), the distance between the minima is the same for both cases, $\Delta \phi=\sqrt{\pi / 4}$, which brings us to the conclusion that the kinks carry fractional charge

$$
Q= \pm \frac{e}{2}
$$

This statement is the main result of the present paper. Three features of the model were necessary to obtain this result: (i) the electron density is bosonized as $\rho=\partial_{x} \phi / \sqrt{\pi}$; (ii) there were two Fermi points with $2 k_{F}=\pi$; (iii) the interaction was strong enough to open the umklapp gap and produce the LRO.

To summarize, we have considered the simplest possible model of interacting electrons subject to an external magnetic field restricting the motion of electrons to hopping on a two-leg ladder. Despite its apparent simplicity, the model exhibits phase transitions to non-trivial LRO phases, the BDW phase in the case $V_{\|}>0$ and the staggered flux phase for $V_{\|}<0$ [19]. In both cases (due to their topological nature) elementary excitations (i.e. kinks) carry fractional charge. As such, charge fractionalization appears to be a generic phenomenon mostly insensitive to the details of the model. As long as the basic features of the model (i.e. the transverse hopping and the flux) are in place any interaction that contains umklapp processes [16] will lead to charge fractionalization.
The applied magnetic field played a crucial role in our considerations. It led to a non-trivial deformation of the single-particle spectrum (Fig. 3) previously unaccounted for and to the appearance of the staggered flux phase. The Fermi momentum became strongly dependent on the flux. For a situation close to half-filling this implies the existence of four essentially different Fermi points. This and other features of the model that were not discussed in the present paper, as well as further generalizations of the model to larger number of chains and spinful electrons is the subject of a future publication [13].

We are grateful to N. Andrei, T. Giamarchi, and A.M. Tsvelik for stimulating discussions.

[1] R. Jackiw and C. Rebby, Phys. Rev. D 13, 3398 (1976); also see A.S. Goldhaber, J. Math. Phys. 44, 3607 (2003).

[2] S.A. Brazovskii, Pis'ma Zh. Eksp. Teor. Fiz. 28, 656 (1978) [JETP Lett. 28, 606 (1978)]; W.P. Su, J.R. Schrieffer, and A.J. Heeger, Phys. Rev. Lett. 42, 1698 (1979); S.A. Kivelson, Synthetic Met. 125, 99 (2001);

[3] G.S. LaRue, W.M. Fairbank, and J.D. Phillips, Phys. Rev. Lett. 42, 142 (1979); V.J. Goldman and B. Su, Science 267, 1010 (1995); M. Reznikov et.al., Nature 399, 238 (1999).

[4] L.N. Cooper, Phys. Rev. 104, 1189 (1956).

[5] R.B. Laughlin, Phys. Rev. Lett. 50, 1395 (1982).

[6] F.H.L. Essler and A.M. Tsvelik, Phys. Rev. Lett. 88, 096403 (2002).

[7] R.S. Eccleston et.al., Phys. Rev. Lett 73, 2626 (1994); M. Azumo et.al., ibid 73, 3463 (1994).

[8] E. Dagotto and T.M. Rice, Science 271, 618 (1996); M. Fabrizio, Phys. Rev. B 48, 15838 (1993).

[9] G. Kotliar, Phys. Rev. B 37, 3664 (1988); I. Affleck and J.B. Marston, ibid 37, 3774 (1988); J.B. Marston and I. Affleck, ibid 39, 11538 (1989).

[10] B.I. Halperin and T.M. Rice, Solid State Phys. 21, 115 (1968); A.A. Nersesyan and G.E. Vachnadze, J. Low Temp. Phys. 77, 293 (1989); A.A. Nersesyan, A. Luther, and F. Kusmartsev, Phys. Lett. A 176, 363 (1993).

[11] For a similar phenomenon in bosonic ladders see E. Orignac and T. Giamarchi, Phys. Rev B 64, 144515 (2001).

[12] I. Affleck in Fields, Strings and Critical Phenomena, North-Holland (1990);

[13] B.N. Narozhny, S.T. Carr, and A.A. Nersesyan, in preparation.

[14] R.E. Peierls, Annales de l'Institut Henri Poincare 5, 177 (1935); R.E. Peierls, Quantum Theory of Solids, Oxford (1955).

[15] L.D. Landau, Z. Phys. 64, 629 (1930).

[16] The transverse interaction does not contribute to the umklapp $g_{2}$ if the coherence factors are approximated by their values at the Fermi points. Since the Taylor expansion of $u$ and $v$ in momentum starts with $k^{2}$, keeping the $k$-dependence would lead to a highly irrelevant umklapp terms. Therefore a naive treatment of the transverse term's contribution to the umklapp by point-splitting would lead to erroneous results because on the lattice this term is not point-split. 
[17] It is common in problems in one dimension to start with a weak coupling and then justify an extension to strong coupling (necessary to reach the gapful phase) a posteriori by comparing with an exact solution [12]. The ladder model is not integrable, however the strong coupling limit of the model shows the same LRO discussed in this paper thus providing us with a justification for our approach.

[18] V.L. Berezinski, Zh. Eksp. Teor. Fiz. 61, 1144 (1971)
[Sov. Phys. JETP 34, 610 (1972)]; J.M. Kosterlitz and D.J. Thouless, J. Phys. C 6, 1181 (1973).

[19] The existence of the two independent interaction constants in the original interaction Hamiltonian Eq. (16) allows us to treat the effective constants $g_{i}$ independently and keep $g_{1}>0$ while allowing $g_{2}$ to become negative. 